\title{
Irreversibility Field in Turbulent Heat Convection Problems
}

\author{
D. Stanciv ${ }^{*}$ and M. Marinescu ${ }^{1}$ \\ 1 Dept. of Engineering Thermodynamics, Politechnica University of Bucharest, \\ Splaiul Independenței 313, sect. 6, Bucharest Romania \\ email: sdorin@mdd.combustion.pub.ro or sdorin_ro@yahoo.com \\ * Corresponding author
}

Résumé - Irréversibilités volumiques dans les processus de convection thermique turbulents Très souvent, les écoulements rencontrés dans les processus de convection thermique utilisés pour produire une puissance mécanique sont turbulents. Pour optimiser ces processus à l'aide du deuxième principe de la thermodynamique, il est nécessaire d'analyser d'abord les mécanismes des irréversibilités. De par la complexité des écoulements turbulents, le niveau global d'analyse ne peut offrir qu'une estimation grossière des dissipations exérgétiques. Le niveau local d'analyse, qui consiste dans le calcul de l'expression de génération volumique d'entropie, a été déjà adapté pour les écoulements turbulents paraboliques, pour lesquels les gradients de la vitesse et de la température moyenne, ainsi que la moyenne des gradients de leurs fluctuations contribuent également aux irréversibilités.

Tenant compte du fait que la première étape du processus d'optimisation thermique, basée sur la minimisation des pertes exergétiques, consiste dans la compréhension des mécanismes de dissipation visqueux et thermique, ce travail est consacré à la simulation mathématique et numérique des irréversibilités volumiques pour quelques problèmes de convection force réalisés par un écoulement turbulent. Les résultats montrent que les mécanismes dissipatifs générés par les fluctuations turbulentes sont aussi importants que ceux issus de gradients de la vitesse de la température moyenne et ils ne peuvent pas être négligés.

Abstract - Irreversibility Field in Turbulent Heat Convection Problems - Most flows occurring in the field of power generation or refrigeration systems are turbulent. The analysis of the turbulent flows irreversibilities represents the first step in the optimization procedures based on the second law of thermodynamics. The simplicity of the irreversibility bulk level analysis cannot offer all the information about turbulence mechanisms of entropy production. The continuum level, which was recently adapted for the turbulent parabolic flows, is by far most sophisticated. Often it is performed only with the aid of numerical simulation, but it can reveal all the peculiarities of entropy generation structure.

Taking into account the importance of entropy creation mechanisms in understanding the second law optimization procedures, this work deal with the theoretical and numerical investigation of irreversibility field for some turbulent heat convection problems. The results show that the irreversibilities created by the turbulent fluctuations are at least as important as those generated by the mean velocity and mean temperature gradients and cannot be neglected. 


\section{LIST OF SYMBOLS}

$b_{x} \quad$ variable flow property defined by eq. (31)

$c_{p} \quad$ specific heat at constant pressure

$c_{v} \quad$ specific heat at constant volume

$C_{\mu} \quad$ constant of K-e model

$C_{f} \quad$ skin friction coefficient

$d_{x} \quad$ variable property defined by the equation (32)

$f_{\mu} \quad$ damping function in the LRN K-e model

$H$ heat convection coefficient

$K \quad$ turbulent kinetic energy

$K_{\theta} \quad$ mean variance of fluctuating temperature

$\mathrm{Pr}$ Prandtl number.

$P_{K} \quad$ production term of $K$

$P_{\theta} \quad$ production term of $K_{\theta}$

Re Reynolds number

$S_{i j} \quad$ strain rate tensor

$\dot{\bar{S}}_{g e n}$ entropy generation rate

$\dot{\bar{S}}_{\text {gen }}^{*}$ dimensionless entropy generation rate

St Stanton number

$u_{i} \quad$ velocity component on the $i$ axis direction

$u_{\tau} \quad$ friction velocity

$u^{+} \quad$ dimensionless velocity in wall coordinate

$T$ absolute temperature

$T_{\tau} \quad$ friction temperature

$T^{+}$dimensionless wall coordinate temperature

$q_{i} \quad$ heat flux vector component on the $i$ axis direction.

\section{GREEK SYMBOLS}

$\alpha \quad$ thermal diffusivity

$\varepsilon_{K} \quad$ dissipation rate of turbulent kinetic energy

$\varepsilon_{\theta} \quad$ dissipation rate of fluctuating temperature variance

$\Phi_{v} \quad$ overall viscous coeficient of irreversibility

$\Phi_{v} \quad$ overall thermal coeficient of irreversibility

$\kappa \quad$ Karman constant

$\lambda$ thermal conductivity

$v \quad$ kinematic viscosity

$\mu \quad$ dynamic viscosity

$\rho$ density

$\tau_{i j} \quad$ stress tensor

$\tau \quad$ time

$\Omega_{i j} \quad$ vorticity tensor.

\section{Subscripts}

$V \quad$ viscous

$T$ turbulent

$$
\begin{array}{ll}
()_{\mathrm{VM}} & \text { viscous mean part } \\
()_{\mathrm{VT}} & \text { viscous turbulent part } \\
()_{\mathrm{QM}} & \text { thermal mean part } \\
()_{\mathrm{QT}} & \text { thermal turbulent part } \\
w & \text { wall. }
\end{array}
$$

\section{Superscripts}

$\bar{\varphi} \quad$ Reynolds mean part of $\varphi$

()$^{(\Omega)}$ volumetric

() linear

()$^{(v)}$ viscous part

( ) $)^{(r)}$ Reynolds part.

\section{INTRODUCTION}

Most flows occurring in the field of power generation or refrigeration systems are turbulent. The understanding of the turbulent flows irreversibilities represents the first step in the optimization procedures based on the second law of thermodynamics.

The irreversibility analysis can be performed at a bulk, or at a continuum level. The bulk level has the advantage of simplicity, but the information about the entropy generation mechanisms is less accurate. The continuum level of analysis is obvious more sophisticated and often it can be performed only by numerical simulation. It always offers not only a great precision of calculus, but also the possibility of a true understanding of the volumetric entropy production structure.

In the case of laminar convection, the continuum level analysis, on which we focus here, shows that the irreversibilities are generated by both viscous and thermal mechanisms, in which the velocity and temperature gradients play the essential role [1]. The turbulence, itself, have to generate its specific mechanisms of irreversibilities because the kinetic exergy of fluctuating velocity and the thermal exergy of fluctuating temperature are also dissipated through the viscosity and the thermal diffusivity of the fluid.

In order to identify these irreversibilities and their magnitude, the expression of the volumetric entropy generation rate will be first averaged in time and next particularized in the case of some turbulent incompressible convection problems.

\section{MATHEMATICAL MODELS}

The mathematical model is splited in two parts, one of them referring to the turbulent flow and another to the volumetric flow irreversibilities. This happens because the volumetric rate calculus of entropy generation needs the properties of the mean flow field, which can be computed with some extensions of the standard two equations $K-\varepsilon$ model. 


\subsection{Mathematical Model of the Flow}

For the incompressible turbulent flow, the Navier-Stokes system contains the Reynolds averaged continuity (1), momentum (2), and energy (3) equations:

$$
\begin{gathered}
\frac{\partial \bar{u}_{\mathrm{i}}}{\partial x_{i}}=0 \\
\frac{\partial\left(\bar{u}_{\mathrm{i}}\right)}{\partial \tau}+\frac{\partial}{\partial x_{j}}\left[\bar{u}_{\mathrm{i}} \bar{u}_{j}+\frac{\bar{p}}{\rho} \delta_{i j}-\frac{1}{\rho}\left(\overline{\tau_{i j}^{(v)}}+\overline{\tau_{i j}^{(r)}}\right)\right]=0 \\
\frac{\partial \bar{T}}{\partial \tau}+\frac{\partial}{\partial x_{i}}\left[\bar{u}_{i} \bar{T}+\frac{1}{\rho c_{p}}\left(\overline{q_{i}^{(v)}}+\overline{q_{i}^{(r)}}\right)\right]=0
\end{gathered}
$$

In the above relations:

$$
\begin{gathered}
\overline{\tau_{i j}^{(v)}}=\mu_{\mathrm{V}}\left(\frac{\partial \bar{u}_{i}}{\partial x_{j}}+\frac{\partial \bar{u}_{j}}{\partial x_{i}}\right) \\
\overline{q_{i j}^{(v)}}=-\lambda_{\mathrm{V}} \frac{\partial \bar{T}}{\partial x_{i}}
\end{gathered}
$$

represent the viscous stress tensor and the heat flux vector of the mean motion field, and:

$$
\begin{gathered}
\overline{\tau_{i j}^{(r)}}=-\rho \overline{u_{i}^{\prime} u_{j}^{\prime}}=\mu_{\mathrm{T}}\left(\frac{\partial \bar{u}_{i}}{\partial x_{j}}+\frac{\partial \bar{u}_{j}}{\partial x_{i}}\right)-\frac{2}{3} \delta_{i j} \rho K \\
\overline{q_{i}^{(r)}}=-\rho c_{p} \overline{u_{i}^{\prime} T^{\prime}}=-\frac{\mu_{\mathrm{T}} c_{p}}{\operatorname{Pr}_{\mathrm{T}}} \frac{\partial \bar{T}}{\partial x_{i}}
\end{gathered}
$$

are the Reynolds stress tensor and the Reynolds heat flux vector, which are related to the mean properties field by the Boussinesq hypothesis. It the above relations $\mu_{\mathrm{T}}, \operatorname{Pr}_{\mathrm{T}}=v_{\mathrm{T}} / \alpha_{\mathrm{T}}$ $=\mu_{\mathrm{T}} c_{\mathrm{p}} / \lambda_{\mathrm{T}}$ and $\lambda_{\mathrm{T}}$ are the turbulent kinematic viscosity, the turbulent Prandtl number, and turbulent thermal conductivity.

In order to close the system (1)-(5), $\mu_{\mathrm{T}}$ and $\lambda_{\mathrm{T}}$ or $\operatorname{Pr}_{\mathrm{T}}$ need to be computed. Taking into account the volumetric irreversibilities occur in the near wall region of the flow [2], the system of equation describing the turbulence must be valid until the solid boundaries. Among the $K-\varepsilon$ models having this feature, the two layer approach of Wolfstein [3] and the low Reynolds number (LRN) formulation of Yang and Shih [4] give best results.

The two layer approach split the wall bounded turbulent flows in two zones. In one of them, located beyond some location situated in the logarithmic sublayer, the equations of standard $K-\varepsilon$ model are used:

$$
\begin{gathered}
\mu_{\mathrm{T}}=\rho C_{\mu} K^{2} / \varepsilon \\
\frac{\partial K}{\partial \tau}+\frac{\partial}{\partial x_{i}}\left[\bar{u}_{i} K-\left(v_{\mathrm{V}}+\frac{v_{\mathrm{T}}}{\sigma_{\mathrm{K}}}\right) \frac{\partial K}{\partial x_{i}}\right]=P_{\mathrm{K}}-\varepsilon
\end{gathered}
$$

$$
\frac{\partial(\varepsilon)}{\partial \tau}+\frac{\partial}{\partial x_{i}}\left[\bar{u}_{i} \varepsilon-\left(v_{\mathrm{V}}+\frac{v_{\mathrm{T}}}{\sigma_{\varepsilon}}\right) \frac{\partial \varepsilon}{\partial x_{i}}\right]=C_{\varepsilon 1} \frac{\varepsilon}{K} P_{K}-C_{\varepsilon 2} \frac{\varepsilon^{2}}{K}
$$

where $P_{K}$ is the production term of $K$. In the wall's vicinity, only the $K$ equation (7) is solved and the turbulent viscosity is computed with the algebraic relation:

$$
\mu_{\mathrm{T}}=\rho C_{\mu} L_{\mu} \sqrt{K}
$$

Additionally, the turbulent dissipation rate results from:

$$
\varepsilon=K^{3 / 2} / L_{\varepsilon}
$$

In the above relations:

$$
\begin{aligned}
& L_{\mu}=\kappa \mathrm{yC}_{\mu}^{-3 / 4}\left[1-\exp \left(-\operatorname{Re}_{\mathrm{y}} / \mathrm{A}_{\mu}\right)\right] \\
& L_{\varepsilon}=\kappa \mathrm{yC}_{\mu}^{-3 / 4}\left[1-\exp \left(-\operatorname{Re}_{\mathrm{y}} / A_{\varepsilon}\right)\right]
\end{aligned}
$$

represent the length scales of $K$ and $\varepsilon$ Theoretical, the surface of racordation is defined as the first location on which the two length scales are equals, but in practice it can be chosen at $y^{+}=150$.

The LRN $K-\varepsilon$ model of Yang and Shih is more elaborated and uses the Kolmogorov time scale as a near wall correction factor for both, turbulent dissipation rate production of equation (8) and Prandtl-Kolmogorov equation (6), which is supplementary corrected with a damping function $f_{\mu}$. As most of the LRN formulation it has the advantage of predicting with more or less success, depending of the flow pattern, the transition phenomena in boundary layer.

\subsection{Mathematical Model of Flow Irreversibilities}

In the incompressible turbulent flow hypothesis, the instantaneous volumetric rate of entropy generation rate is expressed by:

$$
\dot{S}_{g e n}^{(\Omega)}=\left(\dot{S}_{g e n}^{(\Omega)}\right)_{V}+\left(\dot{S}_{g e n}^{(\Omega)}\right)_{Q}
$$

where:

$$
\begin{gathered}
\left(\dot{S}_{\text {gen }}^{(\Omega)}\right)_{V}=2 \frac{\mu_{V}}{T}\left(S_{i j} S_{i j}\right) \\
\left(\dot{S}_{\text {gen }}^{(\Omega)}\right)_{Q}=\frac{\lambda_{V}}{T^{2}}\left(\frac{\partial T}{\partial x_{j}} \frac{\partial T}{\partial x_{j}}\right)
\end{gathered}
$$

represent the viscous and the thermal parts of irreversibilities and $S_{i j}$ denotes the strain rate tensor. Decomposing the flow properties into the mean and the fluctuating part, the Reynolds averaged expression of eq. (12) is [2]:

$$
\dot{\bar{S}}_{\text {gen }}^{(\Omega)}=\left(\dot{\bar{S}}_{\text {gen }}^{(\Omega)}\right)_{V M}+\left(\dot{\bar{S}}_{\text {gen }}^{(\Omega)}\right)_{Q M}+\left(\dot{\bar{S}}_{\text {gen }}^{(\Omega)}\right)_{V T}+\left(\dot{\bar{S}}_{\text {gen }}^{(\Omega)}\right)_{Q T}
$$


The first two terms of the above relation are:

$$
\begin{gathered}
\left(\dot{\bar{S}}_{\text {gen }}^{(\Omega)}\right)_{V M}=\frac{2 \mu_{V}}{\bar{T}} \bar{S}_{i j} \bar{S}_{i j} \\
\left(\dot{\bar{S}}_{\text {gen }}^{(\Omega)}\right)_{Q M}=\frac{\lambda_{V}}{\bar{T}^{2}} \frac{\partial \bar{T}}{\partial x_{j}} \frac{\partial \bar{T}}{\partial x_{j}}
\end{gathered}
$$

and model the viscous and thermal irreversibilities generated in the mean motion field by the gradients of average velocity and average temperature. They are the homologues of the terms modeling the laminar flow irreversibilities because they are generated by the same mechanisms.

The following two terms, containing the correlation of the fluctuating velocity and fluctuating temperature gradients model the proper irreversibilities of the flow turbulence. Thus, the term:

$$
\left(\dot{\bar{S}}_{\text {gen }}^{(\Omega)}\right)_{V T}=\frac{\rho}{\bar{T}}\left(2 v_{V} \overline{S_{i j}^{\prime} S_{i j}^{\prime}}\right)=\frac{\rho \varepsilon}{\bar{T}}
$$

corresponds to the irreversibilities generated by the viscous dissipation $\varepsilon$ of the turbulent kinetic energy $K=\frac{1}{2} \overline{u_{j}^{\prime} u_{j}^{\prime}}$, and the term:

$$
\left(\dot{\bar{S}}_{\text {gen }}^{(\Omega)}\right)_{Q T}=\frac{\lambda_{V}}{\bar{T}^{2}} \frac{\overline{\partial T^{\prime}}}{\partial x_{j}} \frac{\partial T^{\prime}}{\partial x_{j}}=\frac{\rho c_{p}}{\bar{T}^{2}} \varepsilon_{\theta}
$$

characterizes the irreversibilities due to the thermal dissipation $\varepsilon_{\theta}$ of the fluctuating temperature variance $K_{\theta}=\frac{1}{2} \overline{T T^{\prime}}$. As in the previous case, both, the viscosity and the thermal diffusivity of the fluid are involved in the act of entropy generation. Of course, in the equation (18) and (19) the last equalities result from the definitions of $\varepsilon$ and $\varepsilon_{\theta}[5,6]$.

As in the laminar case, the expressions (15)-(19) show that the only sources of the turbulent flow irreversibilities are the viscous and the thermal dissipations. Unlike the laminar flows, the structure of turbulent dissipations is more complicated because they act not only on the averaged exergy part, composed by the mean temperature and mean velocity, but also on the part concerning theirs fluctuating variance.

Knowing the entire structure of volumetric irreversibilities, the entropy generation rate components are computed with:

$$
\left(\dot{\bar{S}}_{g e n}\right)_{V M, V T, Q M, Q T}=\iiint_{\Omega}\left(\dot{\bar{S}}_{g e n}^{(\Omega)}\right)_{V M, V T, Q M, Q T} \mathrm{~d} \Omega
$$

where $\Omega$ is the thermodynamic system's volume. In order to emphasize the importance of flow turbulence irreversibilities we define two overall irreversibility ratios:

$$
\begin{gathered}
\Phi_{V}=\left(\dot{\bar{S}}_{g e n}\right)_{V T} /\left(\dot{\bar{S}}_{g e n}\right)_{V M} \\
\Phi_{Q}=\left(\dot{\bar{S}}_{g e n}\right)_{Q T} /\left(\dot{\bar{S}}_{g e n}\right)_{Q M}
\end{gathered}
$$

Their values depend on the convection geometry as well as on the initial turbulence intensity of the fluid.

\subsection{The Gap Between Models}

Correlating the irreversibility and the flow mathematical models in can be seen that only the first tree components of volumetric entropy generation rate can be computed. For the last one, the mathematical model of the flow does not give any information because its procedure in computing $\lambda_{\mathrm{T}}$ relies on the classical $P r_{\mathrm{T}}=$ const. hypothesis. In this case, the equilibrium turbulence feature of boundary layer can be used, for which the production $P_{\theta}$ and the dissipation rate $\varepsilon_{\theta}$ appearing in $K_{\theta}$ equation are equals, so that:

$$
\left(\dot{\bar{S}}_{\text {gem }}^{(\Omega)}\right)_{Q T} \cong \frac{\rho c_{p} \alpha_{\mathrm{T}}}{\bar{T}^{2}} \frac{\partial \bar{T}}{\partial x_{j}} \frac{\partial \bar{T}}{\partial x_{j}}=\frac{\lambda_{\mathrm{T}}}{\bar{T}^{2}} \frac{\partial \bar{T}}{\partial x_{j}} \frac{\partial \bar{T}}{\partial x_{j}}
$$

It must be emphasize that the above approximation may lead to important errors for some flow configurations. In such a case the two equations $K_{\theta}-\varepsilon_{\theta}$ model [7] could be used.

\section{FROM BULK TO CONTINUUM LEVEL OF IRREVERSIBILITY ANALYSIS}

In order to compare the results of bulk and continuum methods, we consider the forced convection preformed by a fully turbulent flow of an incompressible fluid through a circular straight channel.

For this one-dimensional problem, the global level analysis leads to the following expression of the linear entropy generation rate:

$$
\dot{S}_{g e n}^{(x)}=\left(\dot{S}_{g e n}^{(x)}\right)_{V}+\left(\dot{S}_{g e n}^{(x)}\right)_{Q}=\frac{C_{f}}{2} \frac{\pi \mu_{V}^{3} \operatorname{Re}^{3}}{D^{2} \rho^{2} \bar{T}_{x}}+\pi D h \frac{\left(T_{w}-\bar{T}_{x}\right)^{2}}{\bar{T}_{x} T_{w}}
$$

where $\bar{T}_{x}$ is the bulk temperature of the fluid at the $x$-location, $T_{w}$ stands for the wall temperature, $D$ is the diameter and $h$ denotes the heat transfer coefficient of convection. Of course, the first term of the above relation model the mean and turbulent viscous irreversibilities while the second corresponds to the mean and turbulent thermal ones.

Performing now the continuum level of irreversibility analysis. In the term of wall coordinates, the common dimensionless quantities are:

$$
\begin{gathered}
u^{+}=\frac{u}{u_{\tau}} \\
T^{+}=\frac{T_{w}-T}{T_{\tau}} \\
\varepsilon^{+}=\frac{v \varepsilon}{u_{\tau}^{4}}
\end{gathered}
$$




$$
\varepsilon_{\theta}^{+}=\frac{v \varepsilon_{\theta}}{T_{\tau}^{2} u_{\tau}^{2}}
$$

where $u_{\mathrm{t}}$ and $T_{\mathrm{t}}$ are the friction velocity and the friction temperature. The dimensionless volumetric rate of entropy generation is:

$$
\left(\dot{\bar{S}}_{g e n}^{(\Omega)}\right)^{+}=\dot{\bar{S}}_{g e n}^{(\Omega)} \frac{v T_{w}}{u_{\tau}^{2} \tau_{w}}
$$

Using the classical simplifications for parabolic flows, the volumetric rate components (16)-(19) of entropy generation can be expressed as:

$$
\begin{gathered}
\left(\dot{\bar{S}}_{\text {gen }}^{(\Omega)}\right)_{V M}^{+}=\frac{v_{V}^{+}\left(\partial u^{+} / \partial y^{+}\right)^{2}}{1-b_{x} T^{+}} \\
\left(\dot{\bar{S}}_{\text {gen }}^{(\Omega)}\right)_{Q M}^{+}=\frac{d_{x} \alpha_{V}^{+}\left(\partial T^{+} / \partial y^{+}\right)^{2}}{\left(1-b_{x} T^{+}\right)^{2}} \\
\left(\dot{\bar{S}}_{\text {gen }}^{(\Omega)}\right)_{V T}^{+}=\frac{\varepsilon^{+}}{1-b_{x} T^{+}} \\
\left(\dot{\bar{S}}_{\text {gen }}^{(\Omega)}\right)_{Q T}^{+}=\frac{d_{x} \varepsilon_{\theta}^{+}}{\left(1-b_{x} T^{+}\right)^{2}}
\end{gathered}
$$

where $v_{V}^{+}$stands for the dimensionless kinematic viscosity, $\alpha_{V}{ }^{+}$is the dimensionless thermal diffusivity of the fluid and $b_{\mathrm{x}}$ or $d_{\mathrm{x}}$ are two coefficients containing in theirs definitions the bulk flow properties $\bar{U}$ and $\bar{T}_{x}$, the wall temperature $T_{w}$, the difference $\Delta T_{x}=T_{w}-\bar{T}_{x}$, the skin friction $C_{f}$ and the Stanton number St:

$$
\begin{gathered}
d_{x}=\frac{u_{\tau}^{2}}{c_{p} T_{w}}\left(\frac{\dot{q}_{w}}{u_{\tau} \tau_{w}}\right)^{2}=\left(\frac{S t}{\frac{1}{2} C_{f}}\right)^{2} \frac{\Delta T_{x}}{T_{w}} \frac{c_{p} \Delta T_{x}}{\bar{U}^{2}} \\
b_{x}=\frac{T_{\tau}}{T_{w}}=\frac{S t}{\frac{1}{2} C_{f}} \frac{u_{\tau}}{\bar{U}} \frac{\Delta T_{x}}{T_{w}}
\end{gathered}
$$

The relations (27)-(32) are adaptations of those used in the irreversibility study of turbulent boundary layer [2], because in both cases the inner layer is quite similar. The difference is that the skin friction and the Stanton number, which are related each other by the Colburn analogy, $S t=0.5 C_{f} / \mathrm{Pr}^{2 / 3}$, are both independent from the $x$-direction because of the fully developed turbulence. The same reason makes the derivatives distribution $\partial T^{+} / \partial y^{+}$and $\partial u^{+} / \partial y^{+}$similar along the duct, but the variation of coefficients $d_{x}$ and $b_{x}$, that contain in their expressions the quantity $\Delta T_{x}$, determines the dependence of the flow irreversibilities on the $x$-direction.

Taking into account that $y=R-r$, where $R$ is the duct's radius and $r$ represents the current coordinate, the integration of volumetric entropy rate generation components (27)-(30) lead to the following expressions:

$$
\begin{aligned}
&\left(\dot{\bar{S}}_{\text {gen }}^{(x)}\right)_{V M,(V T)} \cong \frac{1}{8} \frac{\pi \mu_{V}^{3} \mathrm{Re}^{3}}{\rho^{2} D^{2}} \frac{\xi}{\bar{T}} \\
& {\left[\left(1-\frac{\Delta T}{T_{w}}\right) \sqrt{\frac{1}{2} C_{f}} \int_{0}^{\delta_{\text {int }}^{+}}\left(\dot{\bar{S}}_{\text {gen }}^{(\Omega)}\right)_{V M,(V T)}^{+} \mathrm{d} y^{+}\right] } \\
&\left(\dot{\bar{S}}_{\text {gen }}^{(x)}\right)_{Q M,(Q T)} \cong \pi D h \frac{(\Delta T)^{2}}{\bar{T} T_{w}} \frac{S t}{\frac{1}{2} C_{f}} \\
& {\left[\left(1-\frac{\Delta T}{T_{w}}\right) \frac{\sqrt{\frac{1}{2} C_{f}}}{d_{x}} \int_{0}^{\delta_{\text {int }}^{+}}\left(\dot{\bar{S}}_{\text {gen }}^{(\Omega)}\right)_{Q M,(Q T)}^{+} \mathrm{d} y^{+}\right] }
\end{aligned}
$$

From a theoretical point of view, the upper limit of integration should be $R^{+}$, but, based on the constant total shear stress and constant total heat flux assumptions, the derivative's $\partial T^{+} / \partial y^{+}$and $\partial u^{+} / \partial y^{+}$distributions can be computed only on the inner layer, whose dimensionless thickness is $\delta_{\text {int }}{ }^{+}$. This is an acceptable approximation because the turbulent irreversibilities should have small values in the outer layer. In these conditions, the overall irreversibility ratios (21)-(22) can be expressed as:

$$
\begin{gathered}
\Phi_{V}=\int_{0}^{\delta_{\text {int }}^{+}}\left(\dot{\bar{S}}_{g e n}^{(\Omega)}\right)_{V T}^{+} \mathrm{d} y^{+} / \int_{0}^{\delta_{\text {int }}^{+}}\left(\dot{\bar{S}}_{\text {gen }}^{(\Omega)}\right)_{V M}^{+} \mathrm{d} y^{+} \\
\Phi_{Q}=\int_{0}^{\delta_{\text {int }}^{+}}\left(\dot{\bar{S}}_{g e n}^{(\Omega)}\right)_{Q T}^{+} \mathrm{d} y^{+} / \int_{0}^{\delta_{\text {int }}^{+}}\left(\dot{\bar{S}}_{g e n}^{(\Omega)}\right)_{Q M}^{+} \mathrm{d} y^{+}
\end{gathered}
$$

The relations (33) and (34) give the entropy generation rate components, computed with the continuum level of irreversibility analysis. Now, taking into account that:

$$
\left(\dot{\bar{S}}_{\text {gen }}^{(x)}\right)_{V,(Q)}=\left(\dot{\bar{S}}_{g e n}^{(x)}\right)_{V M,(Q M)}+\left(\dot{\bar{S}}_{\text {gen }}^{(x)}\right)_{V T,(Q T)}
$$

the relation (24) and the expressions (33)-(34) will lead to the same results if:

$$
\begin{aligned}
\left(1-\frac{\Delta T}{T_{w}}\right) \sqrt{\frac{1}{2} C_{f}}\left[\int_{0}^{\delta_{\text {int }}^{+}}\left(\dot{\bar{S}}_{g e n}^{(\Omega)}\right)_{V M}^{+} \mathrm{d} y^{+}+\int_{0}^{\delta_{\text {int }}^{+}}\left(\dot{\bar{S}}_{\text {gen }}^{(\Omega)}\right)_{V T}^{+} \mathrm{d} y^{+}\right]=1 \\
\\
\frac{S t}{\frac{1}{2} C_{f}}\left(1-\frac{\Delta T}{T_{w}}\right) \frac{\sqrt{\frac{1}{2} C_{f}}}{d_{x}} \\
{\left[\int_{0}^{\delta_{\text {int }}^{+}}\left(\dot{\bar{S}}_{\text {gen }}^{(\Omega)}\right)_{Q M}^{+} \mathrm{d} y^{+}+\int_{0}^{\delta_{\text {int }}^{+}}\left(\dot{\bar{S}}_{\text {gen }}^{(\Omega)}\right)_{Q T}^{+} \mathrm{d} y^{+}\right]=1 }
\end{aligned}
$$

According to the fully turbulent developed flow hypothesis, $\delta_{\text {int }}{ }^{+}$is constant along the duct's length and have the value $\delta_{\text {int }}{ }^{+}=0.192 \mathrm{R}^{+}[8]$. The results of the calculus for 
$d_{x}=2.0$ and $b_{x}=0.00667$, are presented in Table 1 and the procedure of integration can be found in [2].

It can be seen even for this simple flow that the turbulent dissipations grow up with the Reynolds number and quickly becomes more important than the mean motion ones. In such a condition, for convection geometries involving turbulent wakes, recirculation zones or swirling flows the dissipation is expected to be mainly turbulent.

On the other hand, the numerical data reveal that the differences between the models are less than $5 \%$ in the case of the viscous irreversibilities and less than $12 \%$ in the case of thermal ones.

Taking into account the hypothesis used for deriving the equations (33)-(34) and the approximation (23) utilized for computing the turbulent thermal irreversibilities, the agreement is quite good. This happens because the flow is very simple and properly modeled by the similarity models that allow performing a precise bulk level analysis of irreversibility.

\section{THE CONTINUUM LEVEL OF IRREVERSIBILITY ANALYSIS}

In the case of complex turbulent flows, the bulk level of irreversibility analysis cannot offer a reasonable prediction of entropy generation. Then, the continuum analysis level represents the only choice in the effort of understanding the true mechanism of irreversibility creation and the way in which it is affected by the flow initial parameters and geometry design. The method applicability is next illustrated for two turbulent convection processes: the flows through a corrugated channel and over a staggered tube bank. In the first case, we deal with the turbulent recirculation zones and in the second, additionally, with strong wakes. These kinds of flows appear very often in thermal devices, so that it is relevant to emphasize some peculiarities of theirs irreversibility structure.

In both cases, the mathematical model presented in the second paragraph was used, and the numerical solution was obtained with the aid of commercial software FLUENT 6.0.12.

This solver is based on the finite volume control formulation of the Navier-Stokes equations on an unstructured grid [9]. For the momentum and energy equations, the second order upwind scheme was used and for the pressure correction equation, the PISO algorithm was selected. The numerical solution allowed us to calculate the volumetric irreversibility components, defined by eq. (16)-(19), but for the last one we used the approximation (23). Afterwards, the entropy generation rate was computed with the integral relation (20) and then was put in the dimensionless form with the expression:

$$
\dot{\bar{S}}_{g e n}^{*}=\dot{\bar{S}}_{g e n} /\left(L_{r e f} \rho c_{v} U_{\infty}\right)
$$

where $L_{r e f}$ is a reference dimension and $U_{\infty}$ stands for the inlet velocity.

\subsection{Flow and Heat Transfer Irreversibilities in Corrugated Channels}

The surface corrugation represents a modern technique in the convection heat transfer enhancement. Let consider a bidimensional channel, having only its upper surface sinusoidal

TABLE 1

The results of continuum level on entropy generation analysis for the convection in a straight duct

\begin{tabular}{|c|c|c|c|c|c|}
\hline $\operatorname{Re}$ & $1 \cdot 10^{4}$ & $5 \cdot 10^{4}$ & $1 \cdot 10^{5}$ & $5 \cdot 10^{5}$ & $1 \cdot 10^{6}$ \\
\hline $\int_{0}^{\delta_{\text {int }}^{+}}\left(S_{\text {gen }}^{(\Omega)}\right)_{V M}^{+} d y^{+}$ & 9.059 & 9.146 & 9.158 & 9.169 & 9.171 \\
\hline $\int_{0}^{\delta_{\text {it }}^{+}}\left(S_{g e n}^{(\Omega)}\right)_{V T}^{+} d y^{+}$ & 8.223 & 12.437 & 14.096 & 18.128 & 19.892 \\
\hline$\frac{1}{d_{x}} \int_{0}^{\delta_{\text {mit }}^{+}}\left(S_{g e n}^{(\Omega)}\right)_{Q M}^{+} d y^{+}$ & 7.340 & 7.456 & 7.471 & 7.486 & 7.488 \\
\hline$\frac{1}{d_{x}} \int_{0}^{\delta_{\text {int }}^{+}}\left(S_{g e n}^{(\Omega)}\right)_{Q T}^{+} d y^{+}$ & 4.950 & 8.033 & 9.354 & 12.519 & 13.882 \\
\hline$\Phi_{V}$ & 0.908 & 1.360 & 1.539 & 1.977 & 2.169 \\
\hline$\Phi_{Q}$ & 0.674 & 1.077 & 1.252 & 1.672 & 1.854 \\
\hline Left hand side of eq. (37a) & 0.994 & 0.993 & 0.992 & 0.968 & 0.954 \\
\hline Left hand side of eq. (37b) & 0.886 & 0.896 & 0.902 & 0.893 & 0.888 \\
\hline
\end{tabular}


corrugated. As fluid we chose the air, having at the inlet section the following parameters:

$$
\begin{gathered}
T_{\infty}=350 \mathrm{~K}, \quad T_{w}=300 \mathrm{~K} \\
K_{\infty}=0.003\left(\mathrm{U}_{\infty}\right)^{2}, \quad \varepsilon_{\infty}=1.5\left(K_{\infty}\right)^{3 / 2} / D_{h}
\end{gathered}
$$

At the flow inlet, the fully turbulent profiles for velocity, turbulent kinetic energy and turbulent dissipation rate were used. The Reynolds number of the flow, computed with the mean bulk inflow velocity and the hydraulic diameter of a sinusoidal cell was equal to 7380 . The turbulence was modeled with the two layer approach, and the production term appearing in the turbulent kinetic energy equation (7) was computed with the classical relation, $P_{k}=2 v_{\mathrm{T}} S_{i j} S_{i j}$. In order to obtain the accuracy of numerical solutions, the wall's nearest grid line was set at $y^{+}<1.25$, and the expansion factor of the firsts twelve lines was 1.075 .
Figures 1-4 present the volumetric irreversibility maps of the flow. For the sake of clarity, the greatest values of each irreversibility component were dropped, and the logarithmic scale was chosen for isoline representation. As expected, these figures reveal that the mean motion irreversibilities are concentrated in the near wall region, while the turbulent ones are generated in the entire boundary layer. But as a difference, on the corrugated side of the channel, the acting volume of irreversibility components is also extended within the corrugation and around the interface between the reverse and the bulk flow region. This extension is caused by the eddies which are detached from the wall, near the top of corrugation and are advected in the downstream direction. Because its vorticity, an eddy increases locally the strain rate and the temperature gradient which extract a part of kinetic and thermal energy from the mean flow and transfer it to the fluctuating field. As a consequence, on the detached eddies trajectory

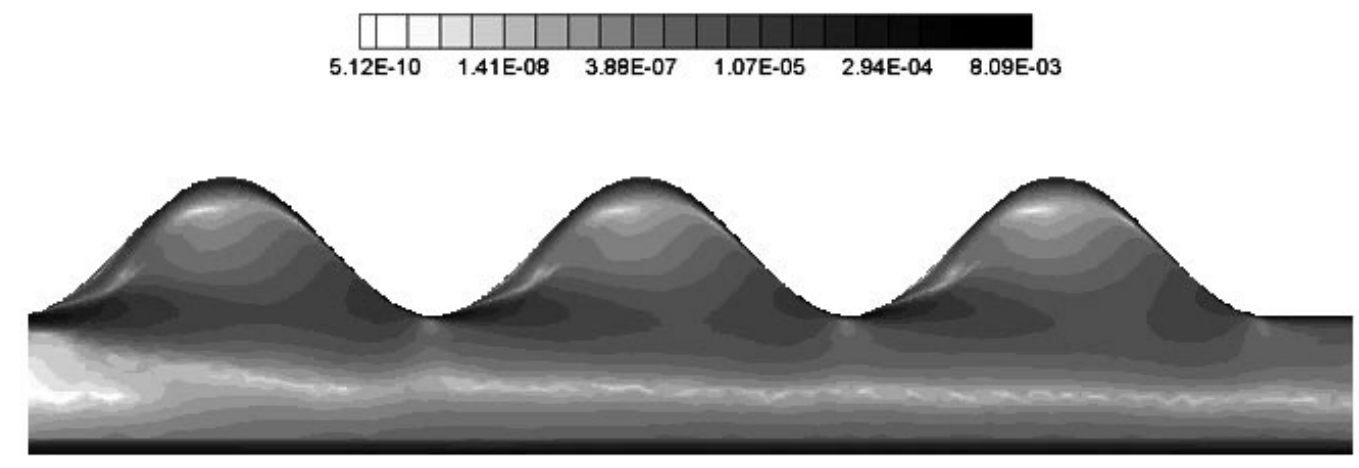

Figure 1

Volumetric mean viscous entropy generation rate for corrugated channel.

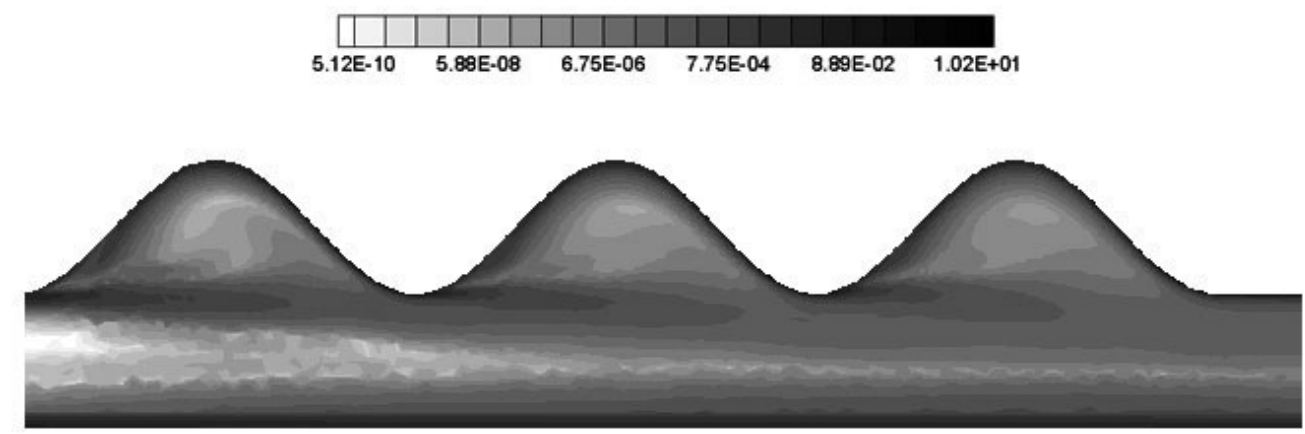

Figure 2

Volumetric mean thermal entropy generation rate for corrugated channel. 


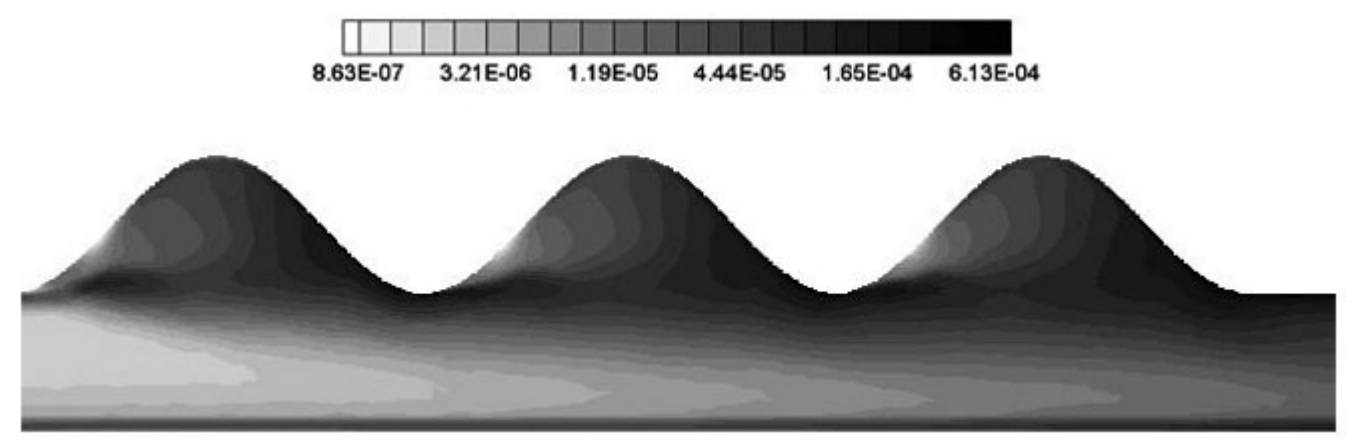

Figure 3

Volumetric turbulent viscous entropy generation rate for corrugated channel.

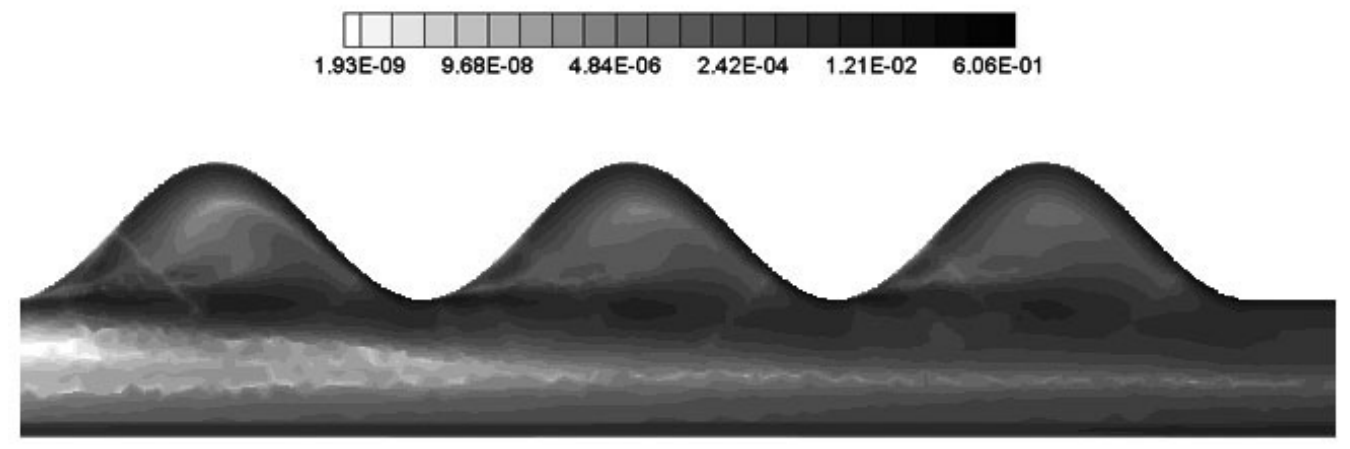

Figure 4

Volumetric turbulent thermalentropy generation rate for corrugated channel.

the irreversibility will be increased not only in its mean part, by the direct growth of mean flow gradients, but also on its turbulent side, by the enhancement of turbulent production. As resulting from Figures 1-4, the latter mechanism is by far most important than the former one because the turbulent production terms $P_{K}$ and $P_{\theta}$ represent the positive part of the sources appearing in $\varepsilon$ and $\varepsilon_{\theta}$ equations.

Another peculiarity of irreversibility maps appears on the corrugated side of the channel. It is generated by the recirculation zone, because around the separation and reattachment points of the flow, the analogy between the velocity and thermal fields no longer holds. In these regions the thermal boundary layer is fully developed, while the hydrodynamic boundary layer is still growing after crossing the stagnation points, so that the thermal components of irreversibility are spread in a greater volume than the corresponding viscous ones. But spreading the thermal irreversibilities in a larger volume does not necessary leads to the growth of entropy production. It seems it is better to have less volumetric irreversibility spreaded in a great space than having great volumetric irreversibility concentrated in a less space.

The map of total volumetric rate of entropy generation is not presented here, but taking into account the above considerations, the total irreversibility distribution is similar to those of mean components near the walls and to those of turbulent components in the bulk flow.

It must be added that the distributions of volumetric irreversibility cannot reveal the relative importance of each component in the budget of entropy production. This happens because the volumetric rate of every component needs to be correlated with its acting volume. For example we can meet very high values of one component distributed in a small volume, or some important values of another one (but not very great), spread in a large volume. So, while the distributions show the places where the volumetric irreversibility is created, the integral values emphasize the contribution of each component in the balance of the entire flow entropy production. 
Table 2 presents in dimensionless form the results of integration for all the volumetric entropy generation rate components on both, the upper (corrugated) and bottom (flat) channel domains. This domains were separated by the median plane of the bulk flow. The turbulent components have by far superior values on the corugated side than on the flat one, because the turbulence levels are higher within the sinusoides than near the smooth boundary. Moreover, heat transfer enhancement increases the mean thermal component of entropy generation rate near the sinusoidal surface by whereas the mean viscous component presents similar values for both domaines. On the other hand, the high level of turbulence is the source of heat transfer enhancement on corrugated surface, so that the greater production of entropy represents the price payed for improving the convection performance.

TABLE 2

Entropy generation rates in corrugated and smooth domains of the channel flow

\begin{tabular}{c|c|c}
\hline$\dot{\bar{S}}_{\text {gen }}^{*}$ & Corrugated domain & Smooth domain \\
\hline$\left(\dot{\bar{S}}_{\text {gen }}^{*}\right)_{V M}$ & $6,297 \cdot 10^{-7}$ & $7,811 \cdot 10^{-7}$ \\
\hline$\left(\dot{\bar{S}}_{\text {gen }}^{*}\right)_{V T}$ & $2,596 \cdot 10^{-6}$ & $2,972 \cdot 10^{-7}$ \\
\hline$\left(\dot{\bar{S}}_{\text {gen }}^{*}\right)_{Q M}$ & $6,033 \cdot 10^{-5}$ & $4,111 \cdot 10^{-5}$ \\
\hline$\left(\dot{\bar{S}}_{g e n}^{*}\right)_{Q T}$ & $6,253 \cdot 10^{-5}$ & $1,228 \cdot 10^{-5}$ \\
\hline$\dot{\bar{S}}_{g e n}^{*}$ & $12,609 \cdot 10^{-5}$ & $5,447 \cdot 10^{-5}$ \\
\hline$\Phi_{V}$ & 4.12 & 0.380 \\
\hline$\Phi_{Q}$ & 1.036 & 0.297 \\
\hline
\end{tabular}

\subsection{Flow and Heat Transfer Irreversibilities for Convection Over a Staggered Tube Bank}

The next application is the irreversibility analysis of the fully turbulent flow over a staggered tube bank. For this case, three basic pattern of flow can be distinguished [11]. If Reynolds number, $R e=\rho U_{\max } D / \mu_{V}$ is less than $10^{3}$, where $U_{\max }$ represents the averaged bulk velocity in the minimum cross section, the flow is laminar. When Reynolds number lies between $10^{3}$ and $10^{5}$, the flow is mixed, because the front of the flow is exposed to a laminar boundary layer and its back to disorderly eddies. So, while the fluid is in turbulent flow through the spaces between tubes, there is a laminar boundary layer developing on the walls and separating at somewhere angular location of tube. But when the Reynolds number exceeds $10^{5}$, the transition in boundary layer occurs, so that the most part of the flow is turbulent.

For our investigation we chose a bank of five staggered tubes, arranged in three columns. Both, the longitudinal and transversal pitch of bank arrangement are equal to 2D. Of course, the flow is periodic on the normal direction to the bulk velocity. In order to obtain the pattern of fully turbulent flow the Reynolds number was set to $1.2 \cdot 10^{5}$. The fluid, which is water, enters in the tube bank with a constant velocity of 5 $\mathrm{m} / \mathrm{s}$, but the maximal bulk velocity of the flow is twice. The water temperature at the inflow boundary is $300 \mathrm{~K}$, while the wall temperature of tubes was set to $340 \mathrm{~K}$. The flow is unsteady but the arrangement of tubes in a staggered bank drastically drops the amplitude of wake oscillations. Besides, from the engineering point of view, the mean temporal field of irreversibility needs to be analyzed. In these conditions, we performed a numerical steady state calculus, which is probable close to the statistic mean of temporal oscillations.

In this case, the LRN $K-\varepsilon$ turbulence model of Yang and Shih was used. As most of the LRN models, it has the ability to predict the flow transition in the boundary layer region. But near the flow stagnation points, the Boussinesq hypothesis fails to simulate the correct level of turbulent kinetic energy, which drastically affects the transition and the heat transfer coefficient prediction. In order to avoid this problem, the production term of $K$ was computed as $P_{K}=2 v_{T} S_{i j} \Omega_{i j}$ [10], where $\Omega_{i j}$ represents the vorticity tensor. This relation was implemented in the solver through an interpreted user defined function [9]. To obtain the numerical solution accuracy, the walls nearest grid line satisfy the condition $y^{+}<1.5 / \mathrm{Pr}^{2 / 3}$, and the expansion factor of the first twenty lines was 1.05. The near wall grid is finer than in previous case because, as compared to air, the greater part of temperature variation takes place in the viscous sublayer.

Let us now analyse the numerical distributions of volumetric irreversibility components, which are plotted in Figures 5-8. As in previous case, some maximal values of each irreversibility component were dropped, so that some little white portions surrounded by isolines correspond to theirs locations.

The distributions of mean viscous and mean thermal irreversibility components are presented in Figures 5 and 6 . As expected, these components act in the near wall regions of the flow and on the trajectories of detached eddies, which are roughly situated at the interface between the recirculation zone and the bulk flow region. Some differences appear on the cylinders back, where the developing process of thermal and hydrodynamic boundary layer after the flow reattachment point is different. From this point of view, there are many similarities with the previous case. Figure 6 shows that the spatial spread of volumetric thermal irreversibility component is not very wide. A high flow rate was imposed at inlet, in order to obtain a great value of Reynolds-number assuring the boundary layer transition before the flow separation. In these conditions, although the Nusselt-number reached an important value, the heat flux transferred from the tubes was not enough to significantly increase the temperature of the bulk flow. On the other hand, because of the water Prandtl-number, the thickness of thermal boundary layer is 


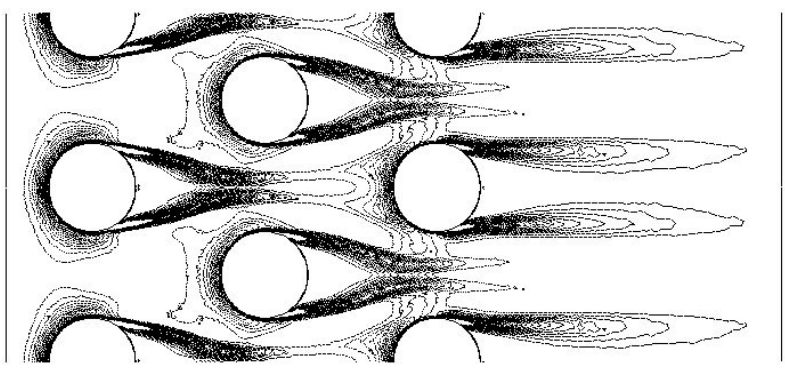

Figure 5

Computed distribution of mean viscous irreversibility component for staggered tube bank.

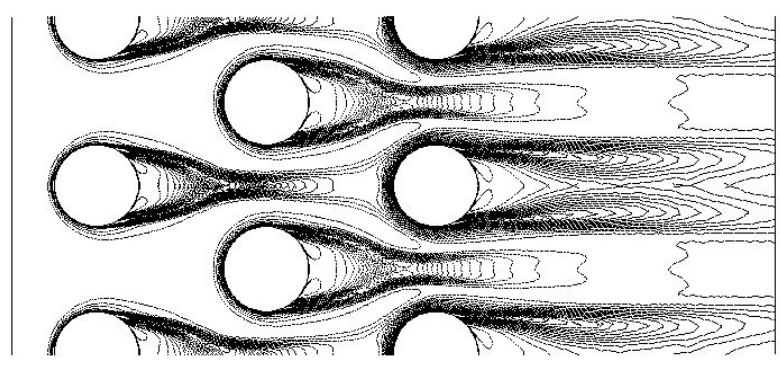

Figure 7

Computed distribution of turbulent viscous irreversibility component for staggered tube bank.

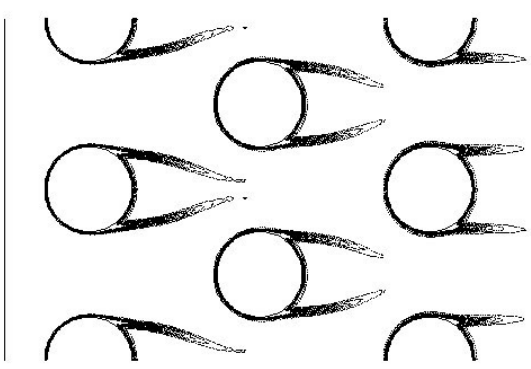

Figure 6

Computed distribution of mean thermal irreversibility component for staggered tube bank.

TABLE 3

Entropy generation rates in staggered tube bank flow

\begin{tabular}{l|c|c|c|c|c|c}
\hline$\left(\dot{\bar{S}}_{\text {gen }}^{*}\right)_{V M}$ & $\left(\dot{\bar{S}}_{\text {gen }}^{*}\right)_{V T}$ & $\left(\dot{\bar{S}}_{\text {gen }}^{*}\right)_{Q M}$ & $\left(\dot{\bar{S}}_{\text {gen }}^{*}\right)_{Q T}$ & $\dot{\bar{S}}_{\text {gen }}^{*}$ & $\Phi_{V}$ & $\Phi_{Q}$ \\
\hline $2.245 \cdot 10^{-6}$ & $1.838 \cdot 10^{-5}$ & $1.984 \cdot 10^{-4}$ & $2.085 \cdot 10^{-4}$ & $4.275 \cdot 10^{-4}$ & 8.187 & 1.051 \\
\hline
\end{tabular}

considerable less than that of the hydrodynamic one. For these reasons, the mean thermal irreversibility got high values, but they were distributed in a small volume.

Figures 7-8 present the distributions of volumetric turbulent irreversibilities, which are created around the tubes and, more important, in the wake regions. Here, theirs values are greatly influenced by the downstream advection of detached eddies which increase the turbulent production terms as well as the turbulent dissipations. Clearly, the wake is the place where the volumetric turbulent components make the rule. As in previous case, we observe a strongly resemblance between the two irreversibility distributions, because both are generated by the same kind of mechanism. Some important values of volumetric turbulent thermal irreversibility occur in the recirculation flow regions, but they are not accompanied by similar levels of the viscous turbulent one.
Table 3 presents the values of dimensionless entropy generation rate components and of the overall irreversibility ratios. As in previous cases, the turbulent components got a surprising important role by comparison with theirs corresponding mean ones. This is also emphasized by the values of overall irreversibility ratios which can be explained by the presence of recirculation zones around which the detached eddies advection enhances the turbulent components of entropy generation. But as usual, the thermal components greatly prevail on the viscous ones. They represent about 95\% from all the flow irreversibilities. Taking into account the hypothesis (23), it seems the temperature gap between the walls and the bulk flow is responsible for this result. In this case, the great difference between $\Phi_{V}$ and $\Phi_{Q}$ is determined not by the small values of turbulent thermal component, but by the high values of mean thermal one. 


\section{CONCLUSION}

The average volumetric irreversibilities of the incompressible turbulent flows are performed by the viscous and thermal dissipations that occur in the mean as well as in the fluctuating part of the motion. The expressions (16)-(19) show that in the both cases the mechanism of gradient interactions is involved in the dissipation process. Thus, the mean motion irreversibilities are created through the interactions of the average velocity or the average temperature gradients, while the turbulent irreversibilities are performed by the interactions of fluctuating velocity or fluctuating temperature gradients.

The continuum level of irreversibility analysis applied to the fully turbulent forced convection through a duct shows that the relations (16)-(19) are in good agreement with the expression (24) derived from the classical bulk level analysis. This happens because the flow is very simple and at a bulk level it is properly modeled by the similarity models.

In the case of complex turbulent flows, only the expressions (16)-(19) can be successfully applied, in addition with the numerical procedures, used for finding the flow and heat transfer solution. Taking into account the mean viscous and mean thermal parts of flow irreversibilities are located in the near wall regions, the mathematical model of turbulence must include those formulations that allow the integration of equations (1)-(5) until the solid boundaries.

The next step in our analysis was the numerical investigation of irreversibility field for two turbulent convection processes: the internal forced convection through a corrugated channel and the external one over a staggered tube bank. The results of numerical simulations revealed the distributions of each irreversibility component and emphasized the great roll of turbulent dissipations in destroying the flow exergy. We also find that, the thermal irreversibilities prevail on the viscous ones, because of the temperature difference between the wall and the inflow fluid.

It is well known the turbulence enhances the heat transfer rates, but as we showed, it also increases the irreversibilities. The high level of entropy production represents the price which must be paid for the heat transfer enhancement. So, between the favorable effects of high turbulence levels and theirs negative aspects it seems to be an optimum, which probably could be find using some economic considerations.
These numerical studies confirmed the utility and the potential of the continuum level method of irreversibility analysis in identifying the flow and heat transfer entropy production that is in correctly predicting the exergy losses. This opens the possibility for numerically optimizing the complex industrial thermal devices. But it cannot be forget the numerical procedure could consume a lot of time because of the very fine grid needed for obtaining a good numerical solution. In these cases, it could be better to get first all the possible informations from the bulk level method and after that, to use them as a starting point for the continuum level of irreversibility optimizations.

\section{REFERENCES}

1 Bejan A. (1988) Advanced Engineering Thermodynamics, John Wiley and Sons, New York 1988.

2 Stanciu, D., Marinescu, M., Isvoranu, D. (2000) Second law analysis of the turbulent flat plate boundary layer. IJAT, 3, 99104.

3 Wolfshtein, M. (1969) The velocity and Temperature Distribution in one Dimensional Flow with Turbulence Augmentation and Presure Gradient, Int. J. Heat Mass Trans, 12, 301-308.

4 Yang, Z., Shih, T.H. (1993) New Time Scale Based K-e Model for Near Wall Turbulence, AIAA Journal, 31, 7, 1191-1198.

5 Tennekes, H., Lumley, J.L. (1972) A First Course in Turbulence, MIT Press, Cambridge, MA.

6 Mohammadi, B., Pironneau, O. (1994) Analysis of the KEpsilon Turbulence Model, John Wiley \& Sons, New York.

7 Sommer, T. P., So, R.M.C., Lay, Y. G. (1997) A near-wall two equations model for turbulent heat fluxes. Int. J. Heat Mass Trans, 12, 3375-3384.

8 Reynolds, A.J. (1974) Turbulent Flows in Engineering, John Wiley \& Sons, New York.

9 Fluent.Inc (2000) User Guide.

10 Launder, B.,E. (1993) Modelling Convective Heat Transfer in Complex Turbulent Flows, in Engineering turbulence modelling and experiments (W. Rodi, A. Martelli editors), Elsevier Science Publishers 3-12.

11 Isacenko, V., Osipova, Y. and Sukomel, A. (1977) Heat Transfer, Editions MIR, Moscow.

Final manuscript received in February 2006

Copyright () 2006 Institut français du pétrole

Permission to make digital or hard copies of part or all of this work for personal or classroom use is granted without fee provided that copies are not made or distributed for profit or commercial advantage and that copies bear this notice and the full citation on the first page. Copyrights for components of this work owned by others than IFP must be honored. Abstracting with credit is permitted. To copy otherwise, to republish, to post on servers, or to redistribute to lists, requires prior specific permission and/or a fee: Request permission from Documentation, Institut français du pétrole, fax. +33147527078 , or revueogst@ifp.fr. 